\title{
Acute Lung Inflammation: Old Illusions of the New Version
}

\author{
Igor Klepikov* MD, \\ Professor, retired. United States \\ *Corresponding Author: Igor Klepikov MD, Professor, retired. United States.
}

\section{Opinion AND PERSPective}

The modern systematic approach to the treatment of acute pneumonia (AP) was formed in recent decades by the use of antibiotics, but the hegemony of antimicrobial principles in the treatment of AP did not immediately arise as a result of the stunning initial effect of penicillin therapy. On the contrary, further multiplication of the role and value of antibiotics in the General medical complex is not entirely logical. The paradox of the situation is that the more antibacterial therapy lost its therapeutic activity, the more significant its perception as the main tool in the treatment of AP became.

It is quite natural that this focus mainly on pathogen suppression has narrowed the range of ideas about the nature of AP and its features. In recent years, the transformation of views on the problem of AP has reached, in my view, the ultimate distortion. Banal forms of bacterial inflammation in the lungs have become regarded as an infectious disease. At the same time, the epidemiology and features of AP have not changed in comparison with those characteristics that have been known to medicine for hundreds of years. Despite the designation of $\mathrm{AP}$ as an infectious process, the recommendations for ensuring epidemiological conditions for this disease remained the same.

At the same time, the value of antibiotics in the treatment of AP has reached its absolute value. In recent years, experts on this problem consider antibacterial therapy as a "cornerstone" in the complex treatment of this category of patients $(1,2)$. No one was confused or discouraged by the fact that the" main medicine " for pneumonia is also the leading means of helping patients with many inflammatory processes that are radically different from AP. In mild forms of the diseases, the whole principle of treatment was actually reduced to the distribution of one drug between different nosological groups of patients. In the case of severe disease, the use of additional funds to help patients with AP did not have significant and specific differences from many other pathologies.

The noted features of the modern AP strategy are well known, as well as the results of its practical implementation, which are reflected in disappointing statistics. For example, in recent years, the death rate from AP among those admitted to intensive care units has reached 36$50 \%$ (3-5). These figures indicate that if the inflammatory process in the lungs requires intensive therapy, then every second or third patient is unsuccessful in such care. Although it is generally recognized that the pathogen of AP remains unknown in most patients, attempts to determine it do not affect the final result, and the choice of antibiotics remains empirical $(2,6)$, treatment failures continue to be explained by the presence of virulent microflora (7). In other words, modern ideas about the nature of AP are frozen on the "microbial" concept of disease, and the lack of arguments in favor of this theory is replaced by unproven assumptions.

Meanwhile, the above statistics are the result of the dominant principles of AP treatment today, which do not take into account a number of fundamental foundations of this disease and, in particular, the unique features of pulmonary circulation. Therefore, as long as approaches to the treatment of AP will repeat the basic principles of care for inflammatory processes of peripheral localization, it is hopeless to expect any success in this direction.

Biased views on the nature of AP and distorted approaches to providing medical care to these patients are becoming more obvious every year. Over time, there are only additional facts and arguments that indicate the need for detailed consideration of all the provisions of the problem. The recent dramatic events caused by the pandemic have quickly exposed many aspects of this problem, not only for specialists, but also for public assessment. Despite the apparent incompatibility between the "usual" forms of AP and coronavirus pneumonia, these lesions have many common features and close 
relationships and, in fact, are variants of the same pathology.

First, the main localization of the lesion in coronavirus infection, as well as the main cause of the severity of the disease and fatal outcomes are viral pneumonia. At the same time, the cardinal sign of this pathology is the defeat of the same organ structures as in bacterial forms of pneumonia (8-10), and a comparison of pathoanatomic findings between these variants of inflammation shows more pronounced changes in the vessels of the small circle in viral lesions $(11,12)$. It is well known that there is a direct relationship between morphology and the function of any organ or tissue in the body. Therefore, localization of pathological tissue transformation is an indirect sign of the identity of pathogenetic mechanisms of disease development, regardless of the type of pathogen.

Second, unlike non-specific bacterial pneumonia, coronavirus lesions differ in their contagiousness and specific pathogen. Despite the presence of a single pathogen, coronavirus infection has an infinite number of manifestations from asymptomatic carrier to the development of terminal conditions. There is no doubt that the clinical manifestation is due to individual characteristics of the body and is similar to the variety of variants of the course of bacterial forms of AP. At the same time, the cause of fatal outcomes in coronavirus infection is not yet explained by the special virulence of the pathogen in tragic cases.

Third, for many years the treatment of acute inflammation in the lungs was based on the etiotropic principle. If these efforts were not effective enough, additional methods of assistance were used. Throughout the entire period of antibacterial therapy, the number of patients with AP who need additional treatment has steadily increased, and among them the most unfavorable results were observed. Automatic transfer of General Therapeutic methods of treatment, such as infusion therapy and medication correction of blood pressure in pathology in the small circle of blood circulation, contradicts fundamental scientific data. However, in discussions on the topic of shock States, patients with AP are analyzed in the General mass of observations along with other nosologies, and non-respiratory lung functions are not even mentioned.

In the course of theoretical discussions, understanding and acknowledging one's own mistakes is usually achieved with great difficulty and long reflection, but the catalyst for this process can be sudden events that change the usual conditions. In this context, such an unexpected phenomenon is the beginning of a pandemic, in which practical medicine received a large influx of patients with viral pneumonia in a short time. Against the background of new medical conditions, the "cornerstone" of traditional treatment has fallen out of the General complex of medical care, and the new situation has shown that among the previously widely used complementary methods of treatment, there were no funds that could really help this severe category of patients.

In the initial stages of viral pneumonia modern medicine does not offer a reliable means of providing care to patients. If a patient with a positive test for coronavirus has respiratory symptoms, then hospitalization for observation and symptomatic means of assistance are recommended, and real support for this category of patients is considered only in case of critical situations in the form of resuscitation methods such as ventilation, positive expiratory pressure, membrane oxygenation $(13,14)$.

The uncertainty of the situation and the impossibility of accurate forecasts even for the near future forces specialists to rely on the availability of breathing equipment and call for an increase in its production $(15,16)$. However, current statistics show that increasing the availability of resuscitation equipment is unlikely to significantly improve results. For example, the overall mortality rate among hospitalized patients with coronavirus infection reaches $26 \%$ (13), but it increases to almost $90 \%$ among patients who were on artificial ventilation (17). Meanwhile, the lack of effective treatment of viral lung lesions is compensated by hopes for success in the development of antiviral drugs, which once again underlines how firmly rooted the foundations of the "microbial" concept of AP are and how these principles determine the future strategy for new conditions of the disease $(18,19)$.

The long-term perception of microbial pathogens of pneumonia as the main and only cause of all complications and treatment failures formed the basis for a persistent selective understanding of the causes and mechanisms of the disease development. These selective ideas about the nature of this nosology continue to determine the vector of search for solutions today and allow us to understand the continuation of the trend to preserve the 
previous set of therapeutic methods. For example, the analysis of treatment of viral pneumonia during the current pandemic, which was conducted by T. M. Rawson et al. (20), showed that bacterial or fungal co-infection was detected in only $8 \%$ of cases, and antibiotics were given to $72 \%$ of patients. Such widespread use of antibiotics reflects the prevailing psychology of views on the problem, and not the result of a balanced professional decision, since the lack of antiviral activity in these drugs is well known, and recommendations against their use in viral lesions remain in force.

Another example of continuing attempts at questionable patient care is the use of methods for replenishing the volume of circulating blood and providing medication for systemic blood pressure. In the context of the current pandemic, the number of cases of AP with bilateral and disseminated forms of inflammation has increased. With a larger volume of lung damage, the number of patients with impaired systemic circulation increases, which is the result of the body's efforts to relieve the small circle of blood circulation and avoid increasing pulmonary edema. Advice on prescribing liquid infusions and using vasopressors in this situation is based on monitoring indicators that are the consequence, not the cause, of current disease mechanisms and reflect only the assumptions of the authors of such recommendations (14). The results of an objective assessment of the effect of infusion therapy on the dynamics of AP are not given, since such studies do not go beyond the monitoring. At the same time, according to analytical reviews, after the start of infusion therapy, many patients with AP have a further decrease in systemic blood pressure, which forces the use of vasopressors and hormones $(2,6,21)$.

The emergence of the coronavirus pandemic is a landmark event that was preceded by unambiguous signals of changing conditions for the development of AP. In the recent past, respiratory viral infections were the causes that prepared the basis for the development of bacterial inflammation (22-24). In the past couple of decades, a number of observed phenomena have clearly demonstrated the ability of viruses to independently cause epidemics of severe forms of pulmonary inflammation. An increase in the number of patients with severe viral pneumonia and an increase in their mortality were observed during the epidemics of SARS, MERS and several flu outbreaks.
Unfortunately, a number of these events, accompanied by a General emotional reaction and justified anxiety, did not have a didactic impact on the system of ideas about the nature of AP and did not lead to a correction of approaches to providing medical care to these patients. Antibiotics, long-term use of which, in my opinion, was one of the leading causes of perestroika among the etiological factors of the disease, continue to be considered as the leading treatment in this group of patients. Modern results of persistent attempts to solve the problem with narrow etiotropic treatment clearly demonstrate the disadvantages of such assistance. Even successful suppression of pathogens does not bring some patients relief from the disease. One example of such dynamics of AP is the well-known observations of the so-called sterile pleural empyema, which emphasize the selective antimicrobial action of antibiotics and their lack of influence on the pathogenetic basis of the inflammatory process.

New circumstances in the context of a pandemic clearly emphasize the importance of pathogenetic mechanisms accompanying acute inflammatory processes in the lungs. Previous experience of applying the etiotropic principle of AP treatment raises serious doubts about the complete cure of patients with coronavirus infection in the case of successful development and use of antiviral drugs. Ways to influence the pathogenetic mechanisms of the disease are a potential option for real improvement of treatment results at the present time.

Confidence in the success of patho genetically based principles of AP treatment stems from their representative testing, the results of which do not depend on time, as they are based on the fundamental materials of medical science (22, 25). Today, fragments of this study have been prepared for public viewing. However, the presence of this data does not mean that it will necessarily be read and bring the desired result. Therefore, continuing efforts to provide medical care to patients with AP, it is necessary to assess in detail the importance of the lungs in the body not only as the main organ of gas exchange. Extra pulmonary lung functions play an important role in regulating homeostasis, and inattention to them can give the wrong direction to therapeutic efforts. The unique anatomical position of the lungs in the General circulatory system and their influence on the Autonomous regulation of blood flow has a special place and crucial importance. Today, in the period of severe clinical situations, information about 
these unique features of the organ should not remain unclaimed.

\section{BIBLIOGRAPHY}

[1] P. Peyrani, L. Mandell, A. Torres, G. S Tillotson (2019) The burden of communityacquired bacterial pneumonia in the era of antibiotic resistance, Expert Review of Respiratory Medicine, 13:2, 139-152, DOI: 10. 1080/17476348.2019.1562339

[2] Weiss S. L., Peters M. J., Alhazzani W. at al. (2020). Surviving Sepsis Campaign International Guidelines for the Management of Septic Shock and Sepsis-Associated Organ Dysfunction in Children, Pediatric Critical Care Medicine: February 2020 - Volume 21 - Issue 2 - p e52e106 doi: 10.1097/PCC.000000000000 2198

[3] A. Liapikou,E. Rosales-Mayor,A.Torres.The management of severe community acquired pneumonia in the ICU. June 2014, Expert Review of Respiratory Medicine 8(3):293303.DOI: $10.1586 / 17476348.2014 .896202$

[4] A. Vidal, L. Santos. Comorbidities impact on the prognosis of severe acute communityacquired pneumonia.Porto Biomedical Journal, Vol. 2. Num. 6.November - December 2017, pages $247-346$

[5] J. W. Kim, J.J. Kim, H. J. Yang at al. The Prognostic Factors of Pneumonia with Septic Shock in Patients Presenting to the Emergency Department. Korean Journal of Critical Care Medicine 2015; 30(4): 258-264

[6] J. P. Metlay, G. W. Waterer, A. C. Long at al.; on behalf of the American Thoracic Society and Infectious Diseases Society of America. "Diagnosis and Treatment of Adults with Community-acquired Pneumonia. An Official Clinical Practice Guideline of the American Thoracic Society and Infectious Diseases Society of America". Amer-ican Journal of Respiratory and Critical Care Medicine, Volume 200, Issue 7, 1 Octo-ber 2019, Pages e45-e67, https://www.atsjournals.org/doi/full/ 10.1164/rccm.201908-1581ST

[7] Schlapbach LJ, Straney L, Alexander J, et al.; ANZICS Paediatric Study Group: Mortality related to invasive infections, sepsis, and septic shock in critically ill children in Australia and New Zealand, 2002-13: A multicentre retrospective cohort study. Lancet Infect Dis 2015; 15:46-54

[8] Hwang, D., Chamberlain, D., Poutanen, S. et al. (2005). Pulmonary pathology of severe acute respiratory syndrome in Toronto. Mod Pathol 18, 1-10 (2005). https://doi.org/10.103 8/ modpathol.3800247

[9] Zhe Xu, Lei Shi, Yijin Wang at al.(2020). Pathological findings of COVID-19 associated with acute respiratory distress syndrome. The Lancet Respiratory Medicine, 2020, Vol 8, Issue 4, P420-422, APRIL 01, 2020
[10] Luo W, Yu H, Gou J, et al . Clinical pathology of critical patient with novel coronavirus pneumonia (COVID-19). Pathology \& Pathobiology 2020:2020020407.

[11] X. M. Ackermann, S. E. Verleden, M. Kuehnel at al. (2020). Pulmonary Vascular Endothelialitis, Thrombosis, and Angiogenesis in Covid-19. May 21, 2020. DOI: 10.1056/NEJMoa2015432

[12] Abernethy K, Sivakumar P, Patrick T, et al. (2020). Coexistent COVID-19 pneumonia and pulmonary embolism: challenges in identifying dual pathology. Thorax. Published Online First: 23 May 2020. doi: 10.1136/thoraxjnl-2020-215 011

[13] Grasselli G et al. (2020). Baseline characteristics and outcomes of 1591 patients infected with SARS-CoV-2 admitted to ICUs of the Lombardy region, Italy. JAMA 2020 Apr 6; [epub]. (https://doi.org/10.1001/jama.2020.5394)

[14] D. A. Berlin, R. M. Gulick, F. J. Martinez (2020). Severe Covid-19. NEJM, May 15, 2020, DOI: 10.1056/NEJMcp2009575

[15] Johns Hopkins Center for Health Security. Ventilator stockpiling and availability in the US. (2020). (http://www. centerforhealths ecurity.org/resources/COVID-19/200214VentilatorAvailability-factsheet.pdf)

[16] M. L. Ranney, V. Griffeth, A. K. Jha (2020). Critical Supply Shortages - The Need for Ventilators and Personal Protective Equipment during the Covid-19 Pandemic. N Engl J Med 2020; 382:e41 DOI: 10.1056/NEJMp2006141

[17] S. Richardson, J. S. Hirsch, M. Narasimhan at al. (2020). Presenting Characteristics, Comorbidities, and Outcomes Among 5700 Patients Hospitalized With COVID-19 in the New York City Area. JAMA. Published online April 22, 2020. doi:10.1001/jama.2020.6775

[18] E. J. Rubin, L. R. Baden, S. Morrissey (2020). Audio Interview: Approaches to Covid-19 Vaccines and Antivirals. N Engl J Med 2020; 382:e58 DOI: 10.1056/NEJMe2012889

[19] A. Bhimraj, R. L. Morgan, A. H. Shumaker at al. (2020). Infectious Diseases Society of America Guidelines on the Treatment and Management of Patients with COVID-19. https://www.idsociety.org/practice-guideline/ covid-19-guideline-treatment-and-management/

[20] Rawson TM, Moore LSP, Zhu N, et al. Bacterial and fungal co-infection in individuals with coronavirus: A rapid review to support COVID-19 antimicrobial prescribing [published online ahead of print, 2020 May 2]. Clin Infect Dis. 2020; ciaa530. doi:10.1093/cid/ciaa530

[21] Alhazzani W et al. Surviving sepsis campaign: Guidelines on the management of critically ill adults with coronavirus disease 2019 (COVID19). Crit Care Med 2020 Mar 27; [e-pub]. (https://doi.org/10.1097/CCM.00000000000043 63) 
[22] Klepikov I. (1989). Acute pneumonia and its purulent and destructive complications in children in the midst of a major industrial centre of Western Siberia. Dissertation for the degree of doctor of medical science. Leningrad. 1989.USSR

[23] Nair GB, Niederman MS (November 2011). "Community-acquired pneumonia: an unfinished battle". The Medical Clinics of North America. 95(6):1143-61.doi:10.1016/j.mcna.2011.08.00 7. PMID 22032432.
[24] Ruuskanen O, Lahti E, Jennings LC, Murdoch DR (April 2011). "Viral pneumonia". Lancet. 377 (9773): 1264-75. doi:10.1016/S0140-67 36 (10)61459-6. PMC 7138033. PMID 21435708.

[25] Igor Klepikov (2019). "The Role and Importance of Biological Stereotypes in the Pathogenesis of Acute Pneumonia." EC Pulmonology and Respiratory Medicine 83 (2019), 239-246.

Citation: Igor Klepikov, “Acute Lung Inflammation: Old Illusions of the New Version”, International Journal of Research Studies in Medical and Health Sciences. 2020; 5(7): 01-05.

Copyright: () 2020 Igor Klepikov, This is an open-access article distributed under the terms of the Creative Commons Attribution License, which permits unrestricted use, distribution, and reproduction in any medium, provided the original author and source are credited. 